\title{
Disjoint multi mobile agent itinerary planning for big data analytics
}

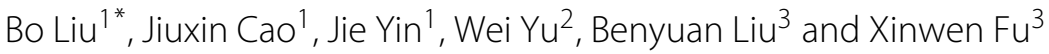

\begin{abstract}
Sensor networks are often part of a cyber-physical system. A large-scale sensor network often involves big data collection and data fusion. The agent technology has drawn much attention in wireless sensor networks (WSNs) to perform data fusion and energy balancing. Existing multi-agent itinerary algorithms are either time-consuming or too complicated in practice. In this paper, we design a routing itinerary planning scheme for the multi-agent itinerary problem by constructing the spanning tree of WSN nodes. First, we build a multi-agent-based distributed WSN (DWSN) model and energy consumption model. Second, we present a novel routing itinerary algorithm named DMAIP, which can group all the sensor nodes into multiple itineraries for agents. We also extend DMAIP and design DMAIP-E, which can avoid long-distance transmission in DMAIP. Our evaluation results demonstrate that our algorithms are better in terms of life cycle and energy consumption than the existing DWSN data collecting schemes.
\end{abstract}

Keywords: Distributed wireless sensor network, Mobile agent, Itinerary planning, Energy efficiency

\section{Introduction}

Wireless sensor networks have been widely used for data collection [1-3] and situation monitoring in various applications, including environment monitoring, automatic target detection, and tracking in battlefields, forests, farmlands, and coal mines. To support these applications, we can place sensors at critical locations. The raw data collected by sensors can then be forwarded through a number of relay nodes to a remote base station, denoted as sink node $[4,5]$. In a WSN, sensor nodes with limited energy are often randomly deployed in massive quantities, and each node may act both as a data collector and a traffic relay, as shown in existing research [4]. Sensor network as a major component of IOT always produces big data [6, 7]. The new techniques make it possible to access more computation power and use more energy; but the energy is still limited, and how to "reduce" the big data is still an important problem in WSN.

The agent-based technology has attracted growing attention to improve energy efficiency, scalability, and reliability of a WSN [8-12]. Agent is now playing a useful role in IOT because it can execute data fusion tasks

*Correspondence: bliu@seu.edu.cn

1 School of Computer Science and Engineering, Southeast University, Nanjing, China

Full list of author information is available at the end of the article autonomously [13], as a result, agent can relieve the network load and prolong lifetime [14] of WSNs. In an agentbased WSN, the main technical challenges include data fusion and energy efficiency, as well as energy and load balancing [15]. For example, Lin et al. [16] leveraged the agent-based technology to balance energy consumption in data collection process of WSNs. Mobile agent-based schemes can also reduce the amount of raw data to be transmitted.

A critical agent-based WSN problem is how to design the itinerary through the WSN for mobile agents (MAs) to collect data. Existing approaches for generating agent itineraries can be classified into two categories: (i) single agent itinerary planning [17-20] and (ii) multi-agent itinerary planning (MIP) [21-23]. For example, Xu et al. [17] investigated static, dynamic, and predictive dynamic schemes to solve the target tracking problem in WSNs. However, the work considers only one target node in the field, their algorithms are based on trilateration, and the itinerary is for only one agent. In reality, using a single agent is not practical because of large delay, unbalanced load, and large accumulated size [21,22]. Therefore, some researchers try to find solutions for MIP. For example, Chen et al. [21] proposed a source-grouping scheme and an iterative MIP algorithm based on single agent itinerary 
planning in order to solve the problem of single agent itinerary planning.

In this paper, we propose a novel multi-agent itinerary planning strategy for WSNs. We also prove that finding optimal balanced disjoint paths for mobile agents to traverse the whole WSN is a NP-hard problem. We design a depth-first-search (DFS)-based itinerary algorithm. We construct the spanning tree over the WSN. Our theoretical analysis shows that our designed algorithm is simple and easy to realize in comparison with existing multiitinerary algorithms. We conduct performance evaluation using QualNet [24] and our results indicate that our novel multi-agent itinerary algorithm achieves better performance than the existing cluster schemes which was always used in cluster-based approaches [12, 25-27]. For example, better energy balancing and longer life cycle can be obtained in our methods.

The multi-agent itinerary planning (MIP) algorithms proposed in this paper are designed for generic large-scale sensor network. We now give a few scenarios to demonstrate its potential practical use. A typical scenario to apply our algorithms is to monitor an environment that is large and dangerous for human being, such as the environment with possible radiation exposure. For example, radiation leak from Tokyo Electric Power's Fukushima Daiichi nuclear power facility contaminated an area of $12.5 \mathrm{mi}$. Large-scale sensor networks can also play an important role in monitoring the areas that are endangered by possible debris flows. In those hostile environments, mobile agents can be dispatched from aircrafts.

A mobile agent is a physical software which can migrate from one sensor to another or from UAVs (or robots) to sensors. To run the mobile agent, some middleware need to be configured which provide the execution environment for agents. Mobile agents can also be applied in visual sensor networks, as illustrated in Fig. 1. As

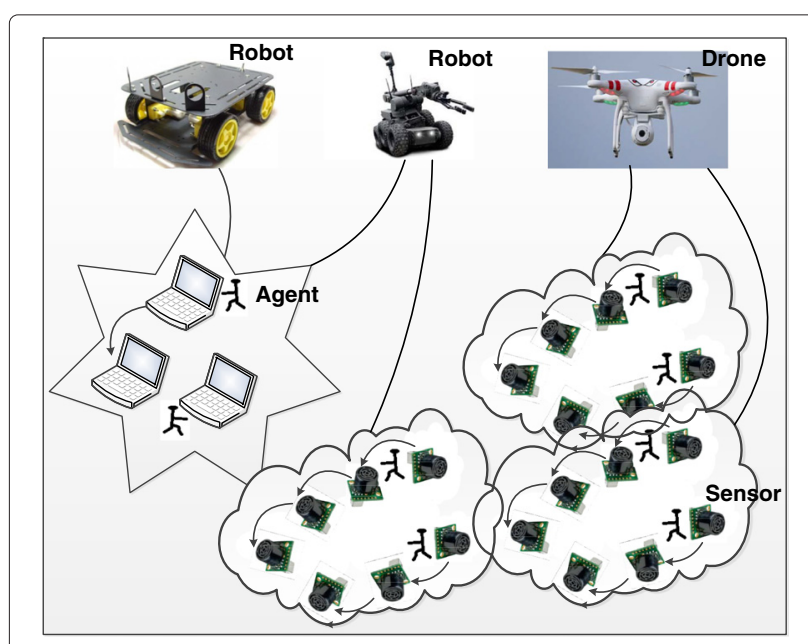

Fig. 1 Mobile agent-based image collection the technology advances, hardware modules are getting smaller. Such small hardware modules can be integrated into sensor devices for specialized use as add-on components. Imagery applications are a classical example. For example, the Cyclops image capturing and inference module can be integrated into popular WSN devices [28]. Mobile agent middleware such as Mobile-C [29] is also available and can be conveniently deployed in sensors, laptops, drones, and other equipments. In Fig. 1, mobile agents carrying image processing codes are dispatched to the target region in order to visit the image sensors according to the arranged itinerary, collecting image data from the corresponding zone of interest. Mobile agents can perform fusion operation over the large volume of imagery data at each sensor node in the target region. Therefore, the mobile agents will be able to migrate, carrying much less load. When the environment of interest changes or the sensing task changes, new mobile agents that carry different image processing algorithms can be dispatched to these image sensors to execute new tasks.

The rest of this paper is organized as follows: In Section 2, we present the most related work. In Section 3, we present two models of agent-based WSNs. In Section 4, we propose the energy consumption model. In Section 5, we present the problem. In Section 6, we present the agent routing approach considering energy balancing. In Section 7, we show performance evaluation results. We conclude the paper in Section 8.

\section{Related works}

In this section, we review the most related work. For a WSN, the problem of multi-agent itinerary planning has attracted growing attention to enhance energy efficiency, scalability, and reliability [21-23]. For example, Chen et al. [21] proposed a source-grouping scheme and an iterative algorithm for multi-agent-based itinerary planning. They partition source nodes into several sets and use the least number of agents while achieving the required coverage of source nodes. However, their idea of selecting the center of area with a high source node density is similar to that of clustering-based method, so a different set of source nodes may cause great difference in numbers. The unbalanced set numbers will lead to the unbalanced task duration of agents and unbalanced energy consumption. Then, Mpitziopoulos et al. [23] began to use a tree structure to compute multi-agents' itinerary. Based on tree structure, they used a greedy-like method to assign itineraries to multi-agents and used mobile agent's cloning capability to clone slave agent at the joint branches. But with the increasing of branches and the increasing number of cloned agents, energy cost also increased largely. Cai et al. [22] applied the genetic algorithm for multiple mobile agents traversing a WSN. Nonetheless, their 
proposed scheme is very complicated and not easy to be implemented.

Clustering is an important strategy to solve the data fusion and collection problem in WSNs and has been widely used. For example, in the conventional low-energy adaptive clustering hierarchy (LEACH) [25] algorithm, the high-energy cluster head positions are randomly rotated so that the energy consumption of sensors can be balanced. In addition, LEACH assumes a homogeneous distribution of sensor nodes in the given area, which may not be realistic. We will compare our algorithm with clustering-based algorithms [26, 27, 30]. It is worth noting that existing research efforts on multi-agent routing mainly focus on how to group source nodes to generate itineraries other than how to compute balanced routes. Our work fills this gap.

\section{Mobile agent-based wireless sensor networks}

In the area of distributed wireless sensor network (DWSN) based on agent, there are commonly two paradigms: (i) single agent-based WSN and (ii) multiagent-based WSN. In the following, we first present the network models for both WSN with single agent and then WSN with multiple agents.

\subsection{Single agent-based WSN}

As shown in the Fig. 2, in a single agent-based WSN, the mobile agent will first move from sink node along paths to the cluster head of a target area and will then collect data. After collecting data from one cluster, the mobile agent will move to the next cluster head and collect data. It will not return to sink node until it reaches the last cluster head. In comparison with the traditional centralized data collection manner (all-to-one), it really reduces network energy consumption to a certain degree. Nonetheless, the process tends to take large time and causes big delay in large-scale WSNs.

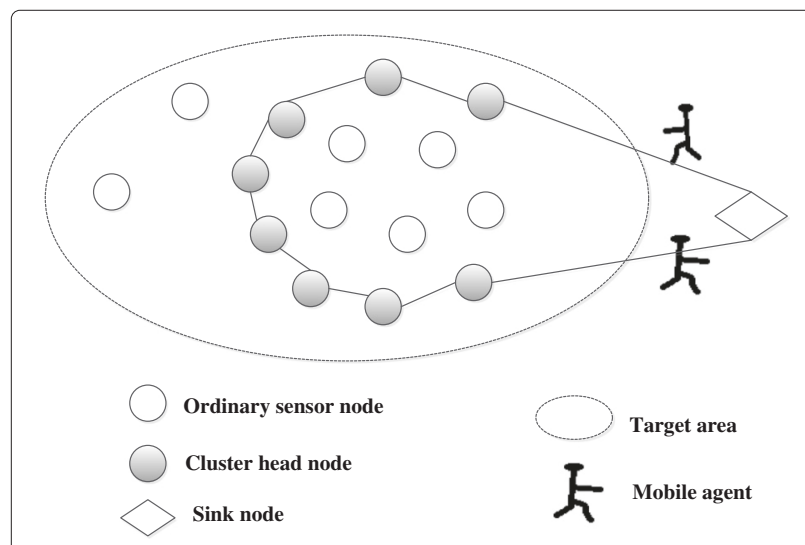

Fig. 2 Single mobile agent data collection model

\subsection{Multi-agent-based WSN}

Based on the single agent model, we propose a multiagent-based data collection scheme. As shown in Fig. 3, there are three components in our scheme: remote user, sink node, sensor node. A remote user assigns tasks to a sink node. All sensor nodes have an operating environment installed for the mobile agent. When the sink node receives a task from a remote user, the sink node traverses network topology to generate a spanning tree, then assigns every path to a mobile agent. When a mobile agent moves along its own path to a sensor node, it will perform the data processing and carry the fused data to the next node. All agents will perform tasks in parallel until they visit all nodes of their paths. The sink node will manipulate data from every path and send back the final results to the remote user. Figure 4 shows the detailed workflow.

\section{Energy consumption model}

We now present the energy consumption model used in our scheme. In a WSN, sensor nodes will communicate with each other through wireless communication. The design of a network topology control algorithm has a great impact on the energy consumption of a sensor node. To the ordinary sensor node, the longer the data transmission distances among sensor nodes are, the more the loss of radio signal is. Facing long-distance data transmissions, we will adopt the method of multi-hop to transmit data in order to save energy.

We adopt the energy consumption model in [31-33] as the single sensor node energy model. The energy consumption for sending data $\left(E_{\mathrm{Tx}}\right)$ consists of the energy consumed by an amplifier circuit ( $\left.E_{\mathrm{Tx} \text {-amp }}\right)$ and by a transmission circuit $\left(E_{\mathrm{Tx} \text {-elec }}\right)$, while the energy consumption for receiving data is composed of the energy consumed by a receiving circuit $\left(E_{\mathrm{Rx} \text {-elec }}\right)$. Based on this, we consider the energy computation for the cluster-based WSN model and the multi-agent-based WSN model in the following.

We consider two modes for energy consumption when sending data: free space transmission mode and multi-hop

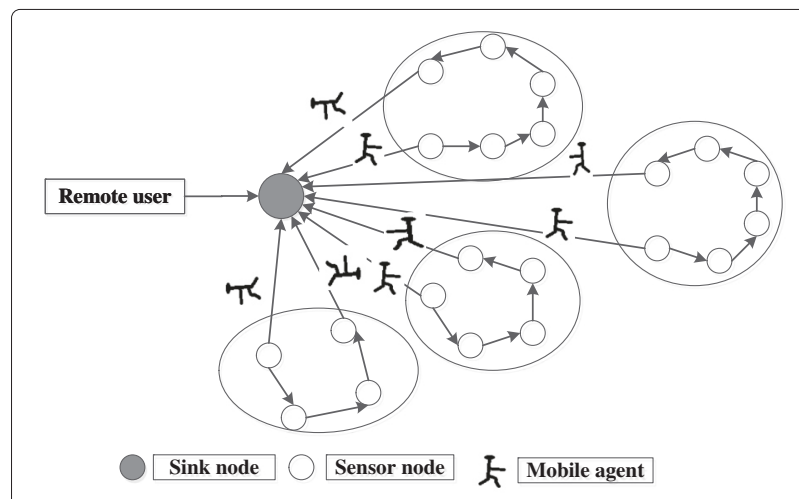

Fig. 3 Multi mobile agent data collection model 


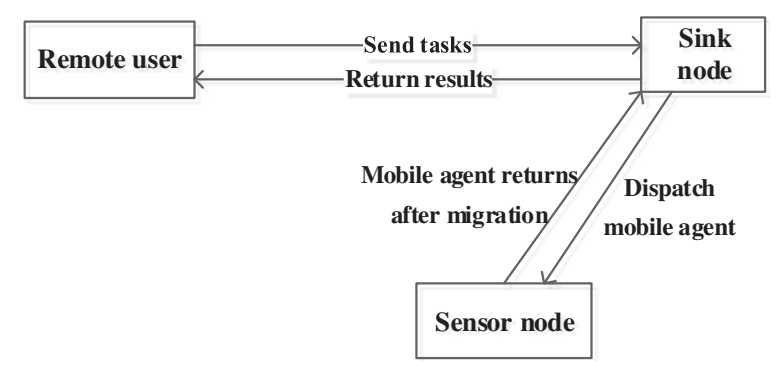

Fig. 4 Task flow model

attenuation mode. The mode that we choose depends on the distance $d$ between the sending node and the receiving node. If $d$ is larger than threshold $d_{0}$, the multi-hop attenuation mode will be chosen. If $d$ is smaller than $d_{0}$, the free space transmission mode will be used. Also, denote $l$ as the size of data packet.

The computational formula of the energy consumption of sending node $\left(E_{T x}\right)$ is

$E_{\mathrm{Tx}}(l, d)=E_{\mathrm{Tx}-\mathrm{elec}}(l)+E_{\mathrm{Tx}-\mathrm{amp}}(l, d)\left\{\begin{array}{l}l E_{\text {elec }}+l \varepsilon_{f \mathrm{~s}} d^{2}, \quad d<d_{0} \\ l E_{\text {elec }}+l \varepsilon_{\text {amp }} d^{4}, \quad d \geq d_{0}\end{array}\right.$

The computational formula of the energy consumption of receiving node $\left(E_{\mathrm{Rx}}\right)$ is

$$
E_{\mathrm{Rx}}(l)=E_{\mathrm{Rx} \text {-elec }}(l)=l * E_{\text {elec }}
$$

In this model, the value of parameter $E_{\text {elec }}$ is the energy consumed by transmitting one bit data; it depends on the methods of channel coding and signal propagation. The typical values of parameters accepted by researchers are

$$
\begin{aligned}
& E_{\text {elec }}=50 \mathrm{~nJ} / \mathrm{bit} \\
& \varepsilon_{\mathrm{amp}}=0.0013 \mathrm{pJ} / \mathrm{bit} / \mathrm{m}^{4} \\
& \varepsilon_{f s}=10 \mathrm{pJ} / \mathrm{bit} / \mathrm{m}^{2}
\end{aligned}
$$

According to [18], given a communication node energy consumption model, the energy consumption consists of three parts: energy for controlling data exchange $E_{\mathrm{Ctr}}$, energy for receiving data $E_{\mathrm{Rx}}$, energy for sending data $E_{\mathrm{Tx}}$. Because every node has the same energy consumption for controlling data exchange, we will ignore it in comparison.

Therefore, the total energy consumption $\left(E_{\text {sum }}\right)$ of every node is

$$
E_{\text {sum }}(l, d)=\left\{\begin{array}{l}
l_{r} * E_{\text {elec }}+l_{t} * E_{\text {elec }}+l_{t} \varepsilon_{f s} d^{2}, \quad d<d_{0} \\
l_{r} * E_{\text {elec }}+l_{t} * E_{\text {elec }}+l_{t} \varepsilon_{\mathrm{amp}} d^{4}, \quad d \geq d_{0}
\end{array}\right.
$$

where $l_{r}$ is the size of received data packet, and $l_{t}$ is the size of transmitted data packet.
Accordingly, we will use the energy formula introduced above to compute the energy consumption of two models: a cluster-based WSN model, a multi-agent-based WSN model.

\subsection{Energy consumption of cluster-based WSN}

In this kind of algorithm such as LEACH algorithm, wireless sensor networks have sink node, cluster head node, and ordinary member node. Here, we assume that cluster head node has ability of fusing data and the rate of fusion is $p$. Ordinary member node sends raw data to its cluster head. Cluster head receives data from every cluster member from two parts: (1) ordinary cluster member nodes and (2) cluster head nodes. Assume that every cluster member node has a data packet whose size is $l$, and that cluster member nodes send data to the cluster head node, and that only sending data consumes energy. From the energy model formula above, we have the energy consumption of cluster member:

$$
E_{\mathrm{mem}}=\left\{\begin{array}{l}
l * E_{\text {elec }}+l \varepsilon_{f_{s}} d^{2}, \quad d<d_{0} \\
l * E_{\text {elec }}+l \varepsilon_{\text {amp }} d^{4}, \quad d \geq d_{0}
\end{array}\right.
$$

Assume every cluster head has $N$ cluster members. The energy consumption of cluster head is divided into two parts: one is for receiving data from cluster members and the other is for sending gathered data to the sink node. So the energy consumption for receiving data $\left(E_{\text {cluRev }}\right)$ will be as follows.

$$
E_{\mathrm{cluRev}}=N * l * E_{\mathrm{elec}}
$$

Energy consumption for sending data $\left(E_{\text {cluTran }}\right)$ is:

$$
E_{\text {cluTran }}=\left\{\begin{array}{l}
{[(N *(1-p)+1)] * l *\left(E_{\text {elec }}+\varepsilon_{f s} d^{2}\right), \quad d<d_{0}} \\
{[(N *(1-p)+1)] * l *\left(E_{\text {elec }}+\varepsilon_{\text {amp }} d^{4}\right), \quad d \geq d_{0}}
\end{array}\right.
$$

We can know that the total energy consumption of cluster head is

$$
E_{\text {clu }}=E_{\text {cluTran }}+E_{\text {cluRev }}
$$

\subsection{Energy consumption of multi-agent-based WSN}

There are only sink node and ordinary sensor nodes in wireless sensor networks. During the process of data collection, a mobile agent will collect and fuse the data of each node. Assume that the size of a mobile agent is $l_{\text {MA }}$, and the size of data packet collected from every sensor node is $l$. Here, we also assume that cluster head node has ability of fusing data and the rate of fusion is $p$. When the mobile agent reaches the $k$ th node, the size of the data in total is

$$
l_{k}=l_{\mathrm{MA}}+[1+(k-1)(1-p)] * l
$$


So the energy consumption of the $k$ th node for sending data is

$$
E_{t}=\left\{\begin{array}{l}
l_{k} * E_{\mathrm{elec}}+l_{k} \varepsilon_{f s} d^{2}, \quad d<d_{0} \\
l_{k} * E_{\mathrm{elec}}+l_{k} \varepsilon_{\mathrm{amp}} d^{4}, \quad d \geq d_{0}
\end{array}\right.
$$

The energy consumption for receiving data is

$$
E_{r}=l_{k-1} * E_{\text {elec }}
$$

So the total energy consumption of the $k$ th node is

$$
E=E_{t}+E_{r}
$$

\section{Problem definition}

Mobile agents can improve data fusion, energy management, and topology management and have attracted great attention in the WSN research. Generally speaking, mobile agents can use raw data on remote sensor nodes, balance the energy consumption of sensor nodes, and improve the reliability by extending the network life cycle.

Given a WSN, we utilize mobile agent techniques to accomplish data collection and fusion with less energy consumption and longer life cycle. A sink node (e.g., base station) is responsible for gathering data from sensor nodes in the WSN. The objective of our research is to find multiple disjoint paths (MDP) with balanced length which cover all sensor nodes in the WSN. The disjoint paths will be assigned to mobile agents.

Definition 1 (MDP problem). Given an undirected connected graph $G=<V, E, W>$, where $V$ is the set of nodes and $E$ is the set of edges, the number of nodes in $V$ is $n, W=\left\{w_{1}, w_{2}, \ldots, w_{m}\right\}$ is a set of weights corresponding to every edge in $E$. The problem is to find $k$ disjoint paths in $G$ to cover all $n$ nodes in $V$, where every edge in these paths can be found in $E$, and to make the longest path (the one with the maximum sum of weights among all the paths) minimized.

In the following, we will prove the MDP problem is NPhard. To prepare for the proof, we firstly give the formal definition of the partition problem which is known as a NP-hard problem.

Definition 2 (Partition problem). Given $n$ positive integers $a_{1}, a_{2}, a_{n}$. The problem is to make the integers be partitioned into two subsets $A$ and $B$ such that the sum of the integers in $A$ equals the sum of the integers in $B$, that is $\sum_{a_{i} \in A} a_{i}=\sum_{a_{j} \in B} a_{j}(1 \leq i, j \leq n)$.

Theorem 1. MDP problem is NP-hard.
Proof. We prove this theorem by constructing a polynomial-time reduction from the partition problem to MDP problem.

Firstly, we create a positive integer $M$ and $M$ is satisfied with the following equation: $M>\sum_{i=1}^{n}\left(a_{i}\right)$. So, we let $M=$ $\sum_{i=1}^{n}\left(a_{i}\right)+1$.

Secondly, For every $a_{i}$, we construct a small undirected weighed graph $G_{i}$ with four vertexes as shown in Fig. 5.

Note that, if this small graph is divided into two parts, then $\alpha$ and $\beta$ must be in the same part; otherwise, there will be one edge with weight $M$ in the one part of the graph.

Thirdly, the small graphs are connected and constructed into a big graph $G^{\prime}$, as shown in Fig. 6 .

From Fig. 6, we can see that the connection between the small graph of $a_{1}$ and the small graph of $a_{2}$ is specially designed as zero weight edges. Obviously, $\alpha_{2}$ and $\gamma_{2}$ must be in two parts, so $\beta_{1}$ will be together with $\alpha_{2}$ in the same part or be together with $\gamma_{2}$ in the same part. The rest can be done in the same manner. As a result, $G^{\prime}$ is divided into two parts, and every part has a path including $2 n$ vertexes whose length is the sum of some integers. The total number of vertexes in $G^{\prime}$ is $4 n$.

The partition problem has the solution if and only if the graph $G^{\prime}$ can be divided into two parts and every part has $2 n$ vertexes; the length of the path passing these vertexes is not more than $\sum_{i=1}^{n}\left(a_{i}\right) / 2$. Here, the $4 n$ is corresponding to the $n$ in the MDP problem; the $2 n$ is corresponding to the $k$ of the MDP problem. So, we have successfully reduced the partition problem to the example of MDP problem in polynomial time. Theorem 1 holds.

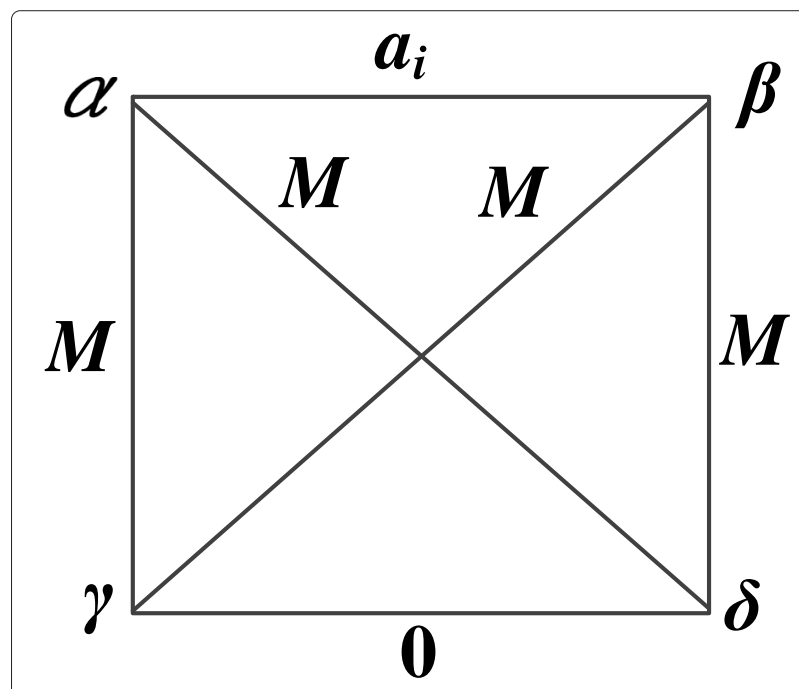

Fig. 5 The construction of $G_{i}$ 


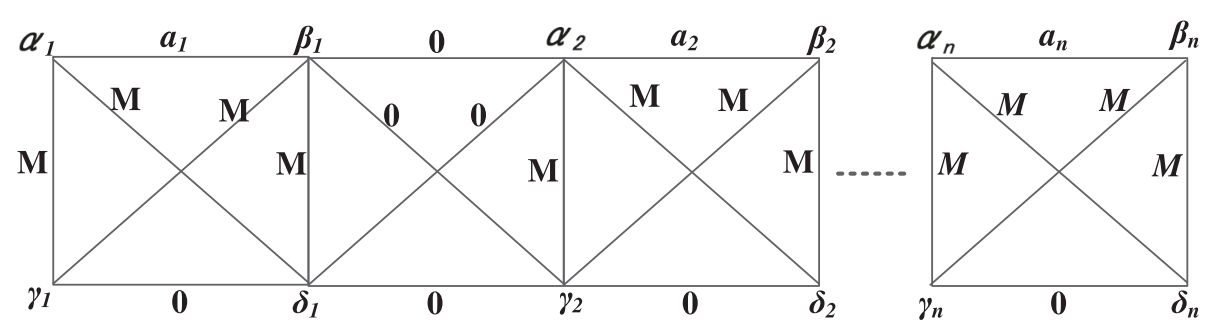

Fig. 6 The construction of $G^{\prime}$

Since it is NP-hard to find optimal number of disjoint paths with balanced length, we focus on developing efficient heuristic and polynomial time algorithms to make agents work effectively in a WSN and produce nearoptimal itineraries.

\section{Algorithms for multi-agent itinerary}

We now present a novel DFS-based multi-agent itinerary planning (DMAIP) algorithm in WSNs. Particularly, we first present the DMAIP algorithm that reduces the communication distance between sensor nodes in the agents' migration path. Then, we present the enhanced DMAIP algorithm, denoted as DMAIP-E. DMAIP-E changes the process of traversing the network, considering the distance between the sink node and sensors when disjoint paths are generated to balance the length of paths.

\subsection{DMAIP algorithm}

In DMAIP algorithm, we first build a network topology graph based on WSN. We then generate a spanning tree of the connected graph in the order of increasing weight and finally traverse the spanning tree recursively to find disjoint paths of all the subtrees.

Algorithm 1 introduces how we build the network topology G. We assume that the wireless sensor nodes can locate themselves. They will send position coordinates to the sink node. Accordingly, the sink node will collect position data and compute distances between all the pairs of nodes. If the distance is not larger than the radius, we leave it as what it is. Otherwise, we will mark it inaccessible explicitly by value -1 .

Algorithm 2 generates a spanning tree of the connected graph G. Basically, we traverse the connected graph or weighted adjacent matrix $M$ by DFS and generate a spanning tree including all nodes in the graph.

In Algorithm 3, we traverse the spanning tree recursively to find disjoint paths of all the subtrees.

There is an example in Fig. 7 to illustrate the generation of paths more clearly. We begin preorder traversal from node $A$, where we get the first path $A-B-C-D-E$. Then, we go back to node $F$, getting the second path $F-G-H$. Then, the third path is $I-J$. The forth path is $K-M$. The last path is $N-O$. As a result, we obtain 5 disjoint paths:

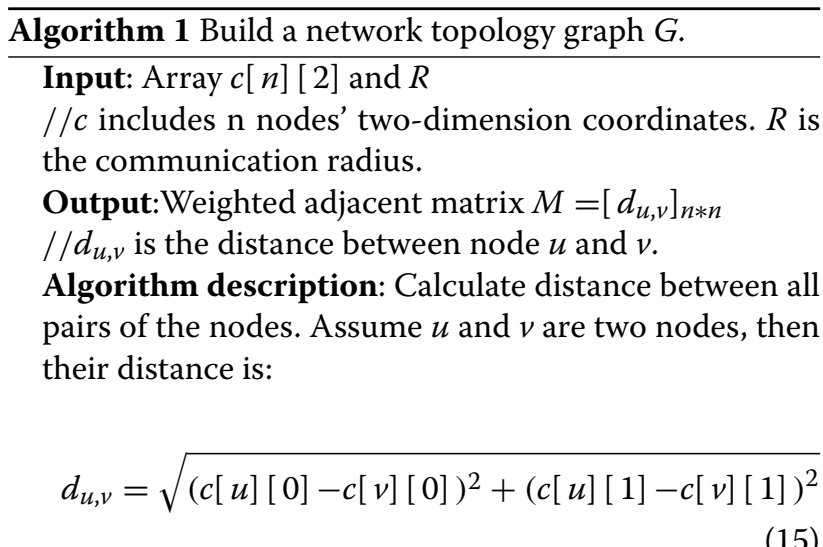

If $d_{u, v} \leq R$ or $u=\operatorname{sink}$ or $v=\operatorname{sink}$, then leave $d_{u, v}$ as what it is, else $d_{u, v}=-1$. If $u=v, d_{u, v}=-1$. It is obvious that $d_{u, v}=d_{v, u}$. At last, we get matrix $M=$ $\left[d_{u, v}\right]_{n * n}$.

(1) $A-B-C-D-E$, (2) $F-G-H$, (3) $I-J$, (4) $K-M$,
(5) $N-O$.

At last, we get disjoint paths including all the sensor nodes in network topology graph. We can send each path to an agent to travel along.

\subsection{DMAIP-E algorithm}

If the sensor node is far away from the sink node, energy consumption increases and this will reduce the lifetime of the WSN. In DMAIP, it is possible to generate such paths that are very long and the end nodes of which may be far away from the sink node. In order to avoid these long paths, we consider the distance between the sink node and sensor nodes during DFS. Based on DMAIP, we propose an algorithm called DMAIP-E that considers the distance between the sink node and all sensor nodes, as shown in Algorithm 4. We first build the network topology graph according to Algorithm 1 of DMAIP to obtain the distance matrix $M=\left(d_{u, v}\right)_{n * n}$. We then generate multiple paths for agents.

In Algorithm 4, we traverse the weighted adjacent matrix $M$ by DFS since the algorithm will generate every 

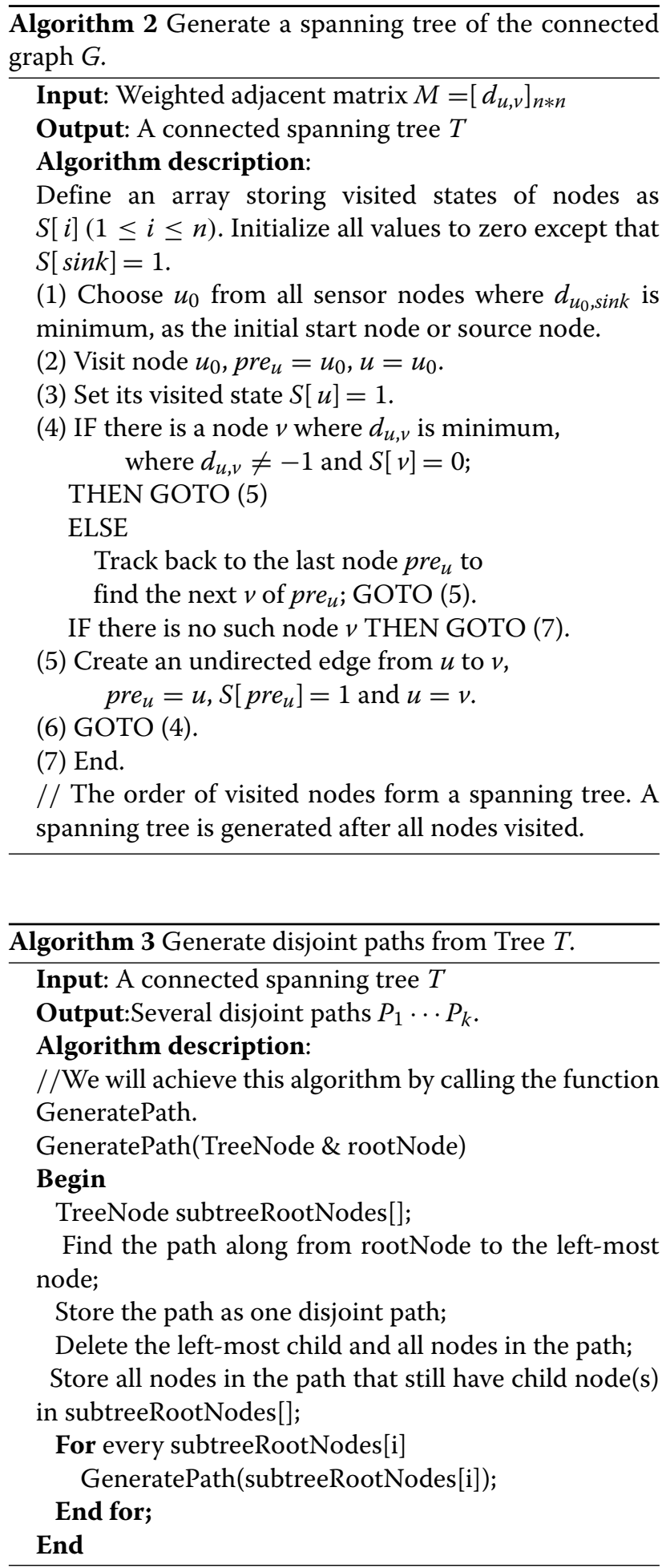

path during this process. We begin with the nearest node $A$ to the sink node; find the next node $B$ that meets Formula 16.

$$
d_{A, \text { sink }}<d_{B, \text { sink }} \quad \text { and } \quad d_{A, B}<d_{B, \text { sink }}
$$

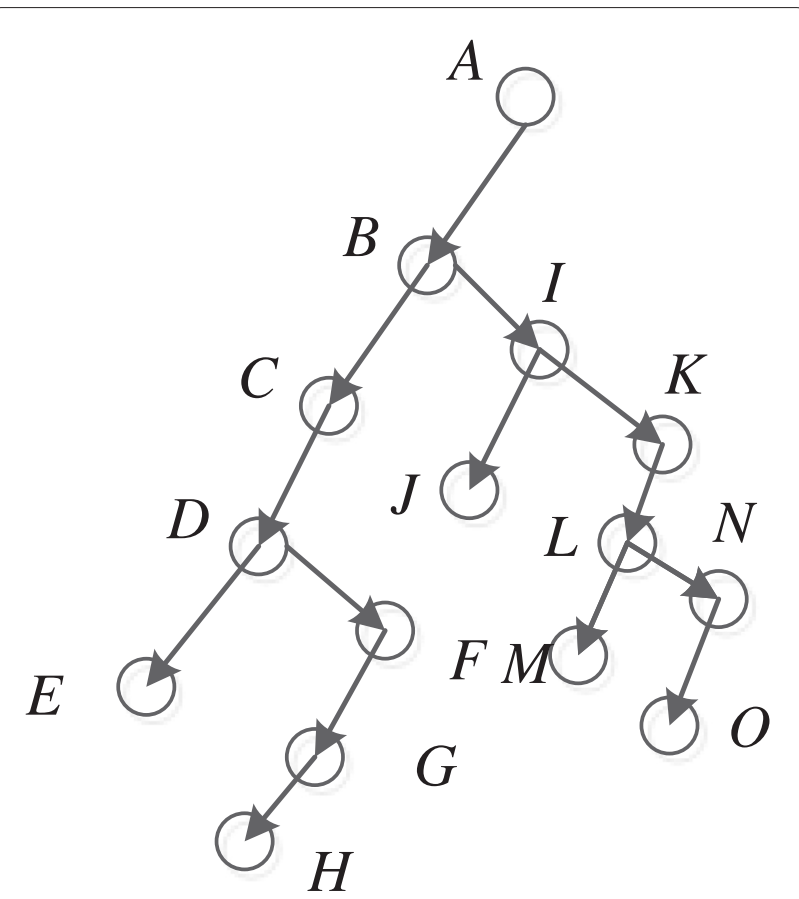

Fig. 7 An example for generation of disjoint path

We illustrate the process of choosing appropriate node $B$ in Fig. 8. Node $B$ must be satisfied with Formula 16. So nodes 1,2 , and 3 are being considered. Besides, we require the nearest node to node $A$. Therefore, node 2 is the node $B$. Assume node $B$ as the next $A$ and find the next node $B$. Once there is no more node $B$ to be found, it will form a path from the first node $A$ to the last node $B$. Then, go

\footnotetext{
Algorithm 4 Generate multiple disjoint paths when traversing.

Input: Weighted adjacent matrix $M=\left(d_{u, v}\right)_{n * n}$

Output:A group of disjoint paths $P_{1} \cdots P_{k}$.

Algorithm description:

Define an array storing visited states of nodes as $S[i](1 \leq i \leq n)$.

Initialize $S[i]$ with 0 except that $S[\operatorname{sink}]=1$.

step (1) Within $M$, find the nearest node $A_{0}$ to the sink node.

$A=A_{0}$

step (2) $S[A]=1$, and then find the appropriate node $B$.

step (3) $A=B, S[A]=1$, and then find the next appropriate node $B$.

step (4) Repeat step (3) until we cannot find the next $B$. Store the path from the first $A$ to the last $B$ as a new path.

step (5) IF there is at least one node $A$ whose state $(S[A])$ is 1 , THEN go to step (2) ELSE end.
} 


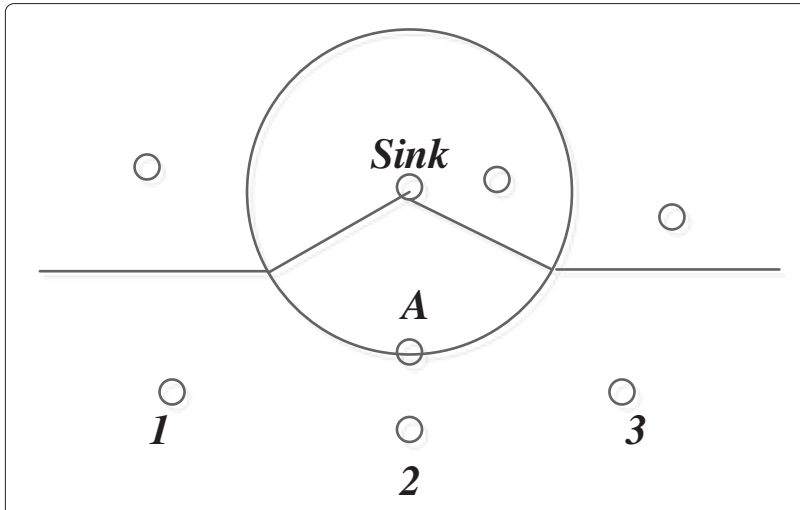

Fig. 8 A process of choosing appropriate node B

back to the sink node and repeat the process above. It will stop when all nodes are included in all generated paths.

The sink node will dispatch an agent to the farthest node of every disjoint path generated above. When an agent finished its task, it will return from the other end of its path to the sink node, and the sink node will manipulate all data from all paths before sending it back to the remote user. In fact, we let the sink node dispatch agents to the outermost location of the path to decrease the distance that the agent loaded with data migrates.

The time complexity of DMAIP algorithm is $O\left(n^{3}\right)$. The time complexity of DMAIP-E algorithm is $O\left(n^{2}\right)$.

\section{Performance evaluation}

We implemented the DMAIP and DMAIP-E schemes in the Qualnet environment [20] and compared them with LEACH algorithm which is the basic of some existing algorithms [26, 27, 30]. We evaluated the performance of the proposed algorithms using the energy model stated in Section 4. Finally, we presented the experimental results of comparing DMAIP, DMAIP-E, and LEACH.

\subsection{Simulation setup}

We deployed sensor nodes randomly in an area of $200 \mathrm{~m} \times 200 \mathrm{~m}$. Each node was deployed at a fixed position. Sink node is located at $(100,100)$. Table 1 gives the parameter settings.

We take the following assumptions: (i) a WSN is of large scale and nodes are distributed randomly, (ii) the initial energy batteries of all the sensor nodes are the same, (iii) the data packet is of the same size, and (iv) all sensor nodes stay in the fixed position.

In this paper, we focused on the data collection process of sensor nodes in WSNs and proposed mobile agent-based schemes to improve the performance of data collection process. We evaluate the performance of our proposed schemes in terms of energy efficiency and life
Table 1 Simulation experimental parameter settings

\begin{tabular}{|c|c|}
\hline Parameter name & Value \\
\hline Number of nodes & $50,75,100,125,150$ \\
\hline Size of the area $/ \mathrm{m}^{2}$ & $200 \times 200$ \\
\hline Location of the base station $/ \mathrm{m}$ & $(100,100)$ \\
\hline Initial energy $E_{\text {init }} / J$ & 0.5 \\
\hline $\begin{array}{l}\text { Energy consumption of transmitting amplifier } \\
E_{\mathrm{fs}} /\left(\mathrm{pJ} * \mathrm{bit}^{-1} * \mathrm{~m}^{-2}\right)\end{array}$ & 10 \\
\hline $\begin{array}{l}\text { Energy consumption of transmitting amplifier } \\
E_{\text {amp }} /\left(\mathrm{pJ} * \mathrm{bit}^{-1} * \mathrm{~m}^{-4}\right)\end{array}$ & 0.0013 \\
\hline $\begin{array}{l}\text { Energy consumption of transmitting and receiving } \\
\text { circuit } E_{\mathrm{elec}} / \mathrm{nJ} * \mathrm{bit}^{-1}\end{array}$ & 50 \\
\hline Initial size of mobile agent /byte & 1024 \\
\hline Length of data packet/byte & 2048 \\
\hline Data fusion rate $p$ & 0.9 \\
\hline Communication radius/m & 40 \\
\hline
\end{tabular}

cycle. In the following, we first compare three related algorithms and then show their performance results.

The clustering mechanism and multi-path mechanism are different representative algorithms for data collection in a WSN. All LEACH-based algorithms use a clustering mechanism for data collection, while our proposed DMAIP and DMAIP-E use multi-path mechanism. The WSN consists of 100 sensor nodes. In our experiments, the three algorithms run in the same environment. Initially, all sensor nodes are deployed as shown in Fig. 9.

Itineraries of LEACH algorithm: In LEACH algorithm, we choose 13 sensor nodes as cluster heads from 100 ones. Ordinary nodes can only communicate with cluster heads,

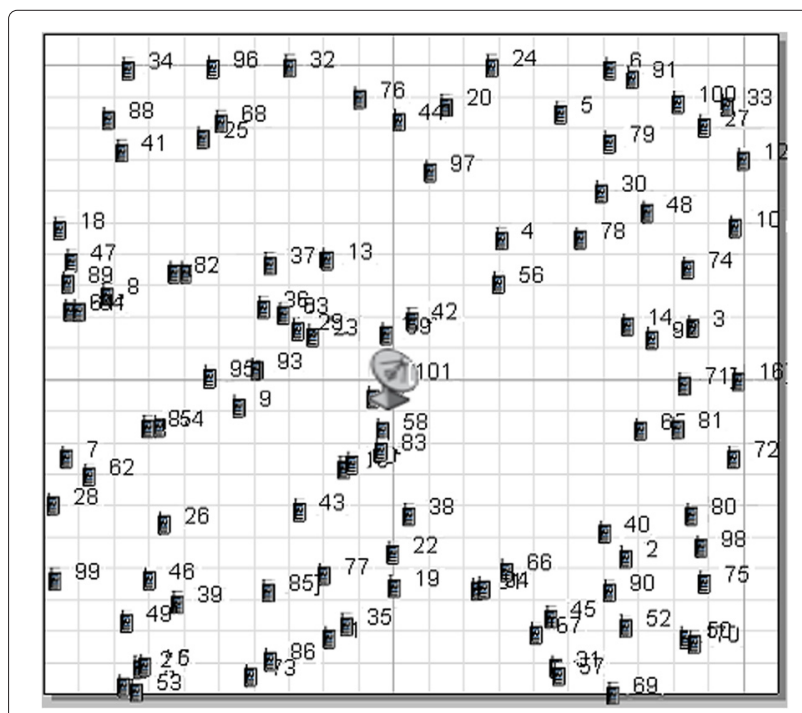

Fig. 9 Deployment of sensor nodes 
and only cluster heads can communicate with sink nodes. Figure 10 illustrates the results for LEACH-based algorithms. According to the LEACH strategy, ordinary nodes will choose the nearest cluster head as theirs because of the randomness of deployed sensor nodes and cluster head nodes. Therefore, each cluster head node manages unbalanced number of ordinary nodes within its domain. For example, there are 2 cluster members in the 86th cluster, while there are 11 cluster members in the 49th cluster. A cluster head may communicates with many cluster members and may have long communication distance with the sink node. This phenomenon leads to a fast death of some cluster heads due to the fast energy consumption. With the increasing number of the sensor nodes in large-scale WSNs, the distance between a cluster head, its cluster member nodes, and sink node will be larger and consume much more energy. Therefore, LEACH is appropriate for relatively small WSNs.

Itineraries of DMAIP and DMAIP-E algorithms: Figure 11 illustrates the results for DMAIP. It has 4 paths and the last node along each path consumes the most energy. For example, in Fig. 11, the 70th node, the last node of a path, is far from the sink node and dies fast because of the fast energy consumption. Figure 12 shows the results for DMAIP-E algorithm. Recall that DMAIP-E algorithm is the enhanced version of the DMAIP algorithm and considers not only the distance between nodes but also the distance between the last node of each path and sink node. The sink node dispatches mobile agents to the farthest end of each path, and the agent returns to the other end of the path. This reduces the amount of energy consumption to a certain degree and extends the life cycle of the wireless sensor network.

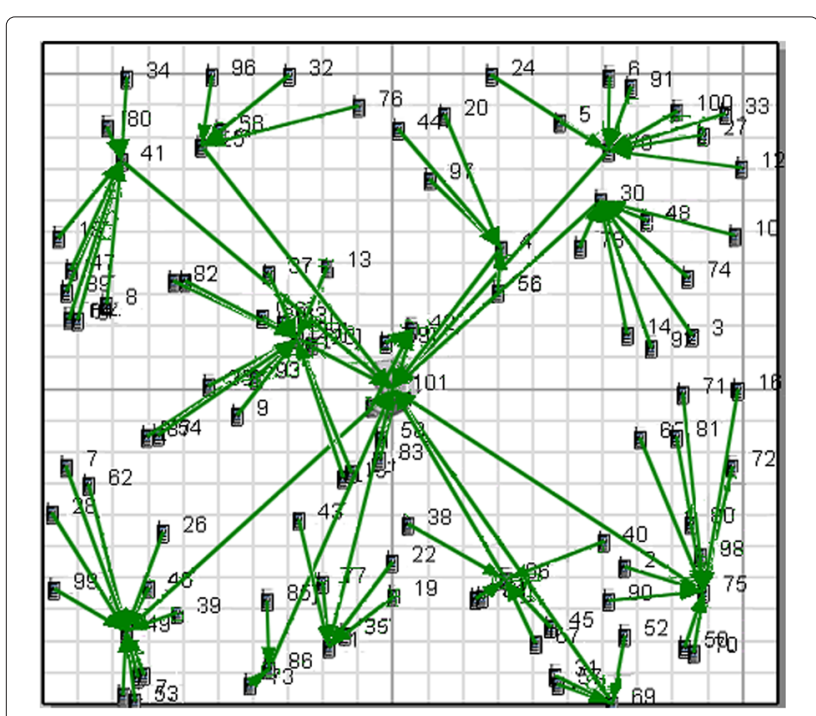

Fig. 10 The branches generated by LEACH

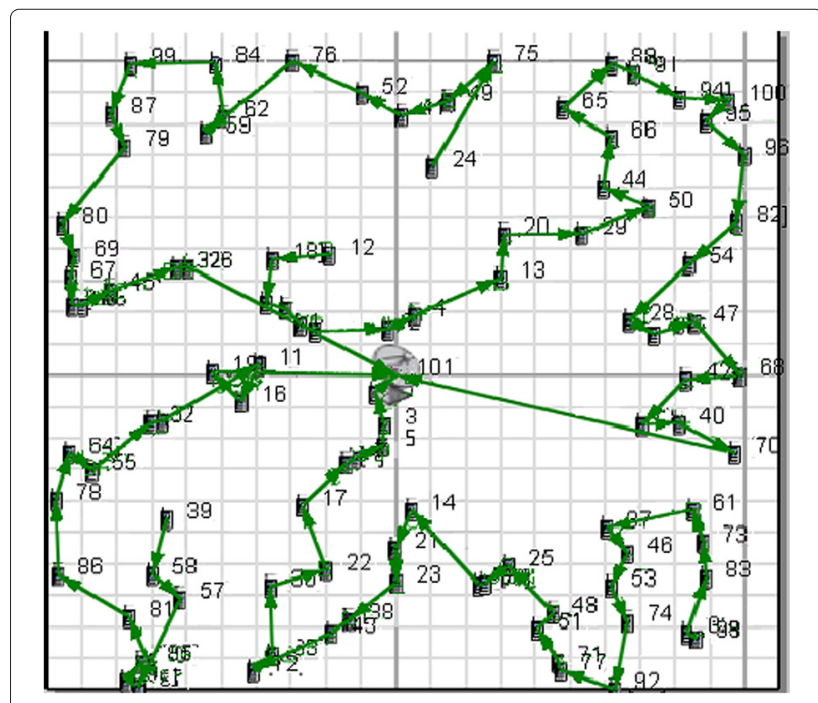

Fig. 11 The branches generated by DMAIP

\subsection{Simulation metrics and results}

To evaluate the performance from our simulation results, the following three performance metrics are considered: life cycle, energy consumption, and impact of the number of nodes. Table 2 gives the evaluation metrics.

Life cycle: From Fig. 13, we can see that with the different number of nodes, the life cycle of DMAIP-E is much longer than that of DMAIP, and the lifetime of DMAIP is a little longer than that of LEACH algorithm as the communication distance of DMAIP-E is the smallest, while the distance of $\mathrm{LEACH}$ is mostly very large.

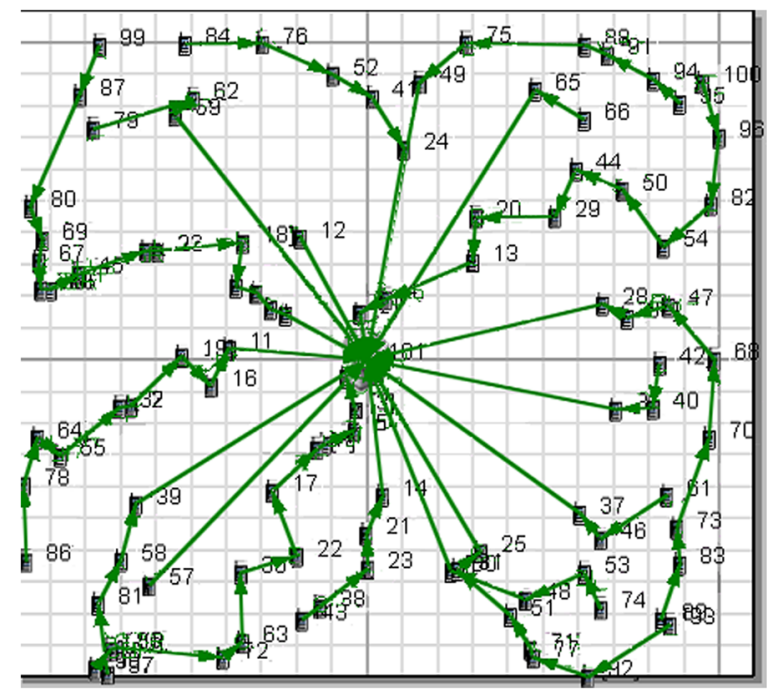

Fig. 12 The branches generated by DMAIP-E 
Table 2 Performance metrics

\begin{tabular}{|c|c|}
\hline Evaluation metrics & Descriptions \\
\hline Round & $\begin{array}{l}\text { The whole process from when the sink } \\
\text { node dispatch agents for every path for } \\
\text { data collection to when all agents return } \\
\text { to the sink node with their data. }\end{array}$ \\
\hline $\begin{array}{l}\text { Energy consumption } \\
\text { of network nodes }\end{array}$ & $\begin{array}{l}\text { The total energy consumption by sensor } \\
\text { nodes when sink node completes one } \\
\text { round of data collection. }\end{array}$ \\
\hline $\begin{array}{l}\text { Number of remaining } \\
\text { nodes }\end{array}$ & $\begin{array}{l}\text { The number of sensor nodes alive in the } \\
\text { network. }\end{array}$ \\
\hline Life cycle & $\begin{array}{l}\text { The round number from the beginning } \\
\text { of the network to the death of the first } \\
\text { node. }\end{array}$ \\
\hline
\end{tabular}

The number of residual nodes: Figure 14 illustrates results, which present the number of remaining nodes versus the number of rounds. As we can see from Fig. 14, as the number of rounds increases, some sensor nodes will die. The number of rounds for LEACH, DMAIP, and DMAIP-E to have dead nodes are 250, 280, and 650, respectively. DMAIP-E has the largest number of rounds, i.e., the longest life cycle, more than twice the rounds of the other two algorithms. The reason why nodes in DMAIP die very early is that it is very far from the end node of some paths to the sink node and therefore transmitting data from the end node to the sink node will consume energy heavily. In addition, all nodes for LEACH die in about 600th round, while for DMAIP and DMAIP$\mathrm{E}$, nodes will not be all dead until about 1500th round. This is because DMAIP and DMAIP-E adopt a mobile agent model, which could save and balance the energy of sensor nodes. Also, DMAIP-E outperforms other schemes.

Energy consumption: Energy consumption is another important performance metric for WSNs. This experiment was performed in a network with 100 nodes, and

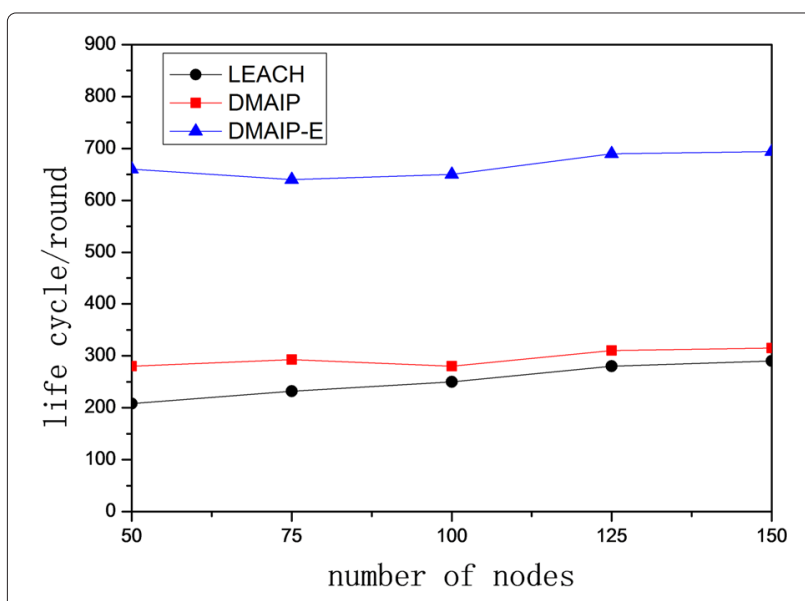

Fig. 13 Life cycle with different number of nodes

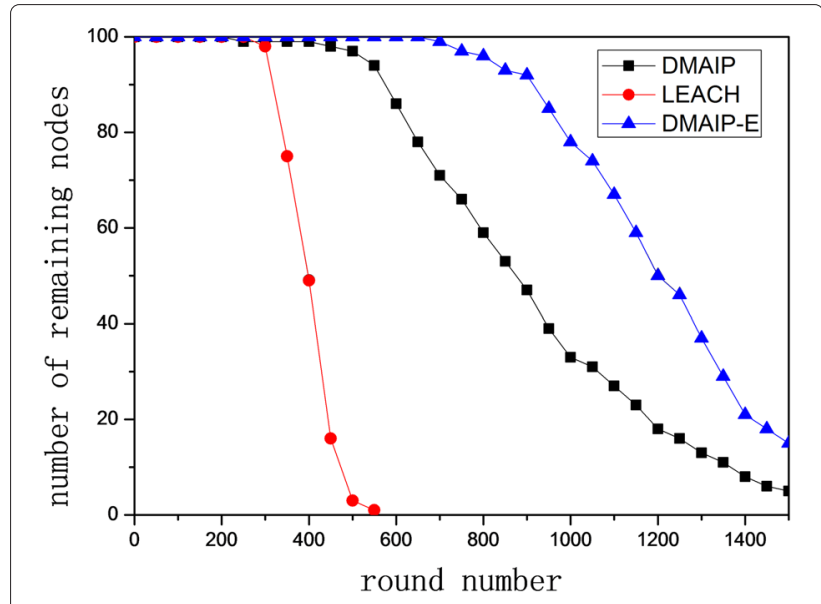

Fig. 14 Changes of residual nodes' number with rounds increasing

the results are shown in Fig. 15. As we can see from the figure, for every round, the energy consumption of DMAIP and DMAIP-E algorithm is almost the same while the energy consumption of LEACH is not stable. This is because DMAIP and DMAIP-E collect data according to preset paths while LEACH chooses cluster heads randomly and changes it every few rounds. Therefore, energy consumption of LEACH is different in different round. The energy consumption of $\mathrm{LEACH}$ is not stable because clusters are randomly formed without periodic mode. In every round, DMAIP-E algorithm consumes the least energy while LEACH uses the most.

\section{Conclusions}

The itinerary planning problem is a key issue in distributed WSNs. In this paper, we presented a multi-agentbased paradigm for data collecting in WSN. Because the optimal multi-agent itinerary generation problem is

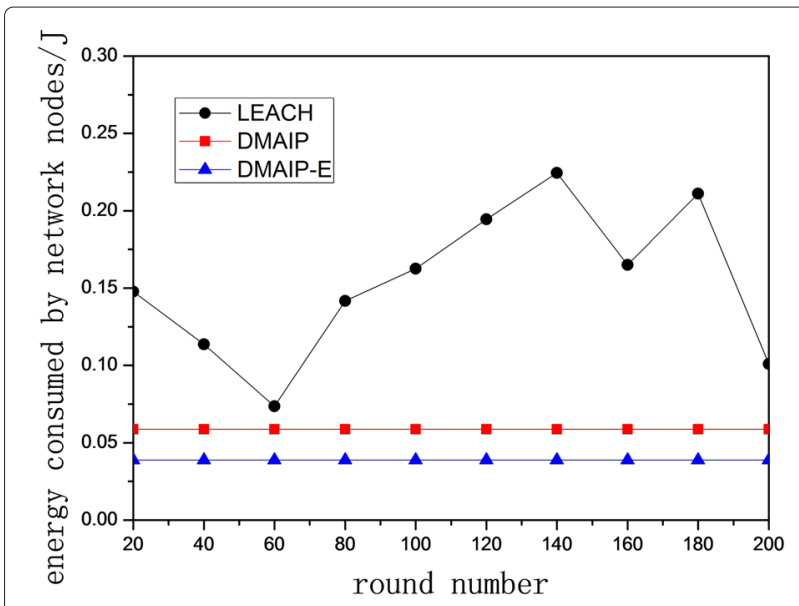

Fig. 15 Total energy of nodes in different round 
NP-hard, we proposed an approximate algorithm called DMAIP-E based on the global topology graph. Our simulation results show that DMAIP-E is appropriate for large-scale WSN and has better performance than existing clustering-based algorithms in terms of both life cycle and energy consumption.

\section{Competing interests}

The authors declare that they have no competing interests.

\section{Acknowledgements}

This work is supported by the National Natural Science Foundation of China under Grant Nos. 61370208, 61472081, 61320106007, and 61272531, China National High Technology Research and Development Program (2013AA013503), NSF under award CNS-1527303, SGCC Science and Technology Program "key technologies and application of test platform for dispatching automation system", Collaborative Innovation Center of Social Safety Science and Technology, Collaborative Innovation Center of Wireless Communications Technology, Jiangsu Provincial Key Laboratory of Network and Information Security (BM2003201), and Key Laboratory of Computer Network and Information Integration of Ministry of Education of China under Grant No. 93K-9.

\section{Author details}

${ }^{1}$ School of Computer Science and Engineering, Southeast University, Nanjing, China. ${ }^{2}$ Department of Computer and Information Sciences, Towson University, Baltimore, USA. ${ }^{3}$ Department of Computer Science, University of Massachusetts Lowell, Lowell, USA.

\section{Received: 24 November 2015 Accepted: 4 April 2016}

Published online: 12 April 2016

\section{References}

1. $\mathrm{S} \mathrm{Ji}, \mathrm{Z}$ Cai, Distributed data collection in large-scale asynchronous wireless sensor networks under the generalized physical interference model. IEEE Trans. Netw. 21(4), 1270-1283 (2013)

2. S Chen, M Huang, S Tang, Y Wang, Distributed data collection in large-scale asynchronous wireless sensor networks under the generalized physical interference model. IEEE Trans. Parallel Distributed Syst. 21(4), 52-60 (2012)

3. S Ji, R Beyah, Z Cai, Snapshot and continuous data collection in probabilistic wireless sensor network. IEEE Trans. Mobile Comput. 13(3), 626-637 (2014)

4. F Wang, D Wang, JC Liu, in Proceedings of the 6th Annual IEEE Communications Society Conference on Sensor, Mesh and Ad-HoC Communications and Networks. Traffic-aware relay node deployment for data collection in wireless sensor networks, (Rome, Italy, 2009), pp. 351-359

5. H Luo, Y Liu, SK Das, Distributed algorithm for en route aggregation decision in wireless sensor networks. IEEE Trans. Mobile Comput. 8(1), $1-13$ (2009)

6. A Zaslavsky, C Perera, D Georgakopoulos, in Proceedings of the International Conference on Advances in Cloud Computing, ACC 2012. Sensing as a service and big data, (Bangalore, India, 2012), pp. 21-29. http://citeseerx.ist.psu.edu/viewdoc/summary?doi=10.1.1.294.9753

7. T-M Choi, HK Chan, X Yue, Recent development in big data analytics for business operations and risk management. IEEE Trans. Cybernet. 2016(99), 1-12 (2016)

8. H Qi, X Wang, SS lyengar, K Chakrabarty, in Proceedings of the 5th International Conference on Information Fusion. Multisensor data fusion in distributed sensor networks using mobile agents, (Singapore, 2001), pp. 11-16. http://citeseerx.ist.psu.edu/viewdoc/summary?doi=10.1.1.10 7251

9. W Du, J Deng, YS Han, PK Varshney, in Proceedings of the 10th ACM conference on Computer and communications security (CCS 2003). A pairwise key pre-distribution scheme for wireless sensor networks, (Washington, DC, USA, 2003), pp. 42-51. http://dl.acm.org/citation.cfm? id $=948118$
10. L Eschenauer, VD Gligor, in Proceeding of the 9th ACM Conference on Computer and Communications Securit (CCS '02). A key-management scheme for distributed sensor networks, (Washington, DC, USA, 2002), pp. 41-47. http://dl.acm.org/citation.cfm?id=586117

11. $\mathrm{H}$ Qi, $, Y X u, X$ Wang, Mobile-agent-based collaborative signal and information processing in sensor networks. Proc. IEEE. 91(8), 1172-1183 (2003)

12. E Amiri, H Keshavarz, AS Fahleyani, H Moradzadeh, S Komaki, in IEEE International Conference on Space Science and Communication (IconSpace 2013). New algorithm for leader election in distributed WSN with software agents, (Melaka, Malaysia, 2013), pp. 290-295. http://ieeexplore.ieee.org/ xpls/abs_all.jsp?arnumber $=6599483$

13. L Jarvenpaa, M Lintinen, A-L Mattila, in 17th International Conference on System Theory, Control and Computing (ICSTCC). Mobile agents for the internet of things, (Sinaia, Romania, 2013), pp. 763-767. http://ieeexplore. ieee.org/xpls/abs_all.jsp?arnumber $=6689053$

14. J Chen, S He, Y Sun, et al., Optimal flow control for utility-lifetime tradeoff in wireless sensor networks. Comput. Netw. 53(18), 3031-3041 (2009)

15. C Wu, GS Tewolde, W Sheng, B Xu, Y Wang, Distributed multi-actuator control for workload balancing in wireless sensor and actuator networks. IEEE Trans. Automatic Control. 56(10), 2462-2467 (2011)

16. K Lin, M Chen, S Zeadally, JJPC Rodrigues, Balancing energy consumption with mobile agents in wireless sensor networks. Future generation computer systems. Future Generation Comput. Syst. 28(2), 446-456 (2012)

17. $\mathrm{YXu}, \mathrm{H} \mathrm{Qi}$, Mobile agent migration modeling and design for target tracking in wireless sensor networks. Ad Hoc Netw. 6(1), 1-16 (2008)

18. M Chen, L Yang, T Kwon, L Zhou, Itinerary planning for energy-efficient agent communications in wireless sensor networks. IEEE Trans. Vehic. Technol. 60(7), 3290-3299 (2011)

19. B Chen, W Liu, Mobile agent computing paradigm for building a flexible structural health monitoring sensor network. Computer-aided Civil Infrastruct. Eng. 25(7), 504-516 (2010)

20. Q Wu, NSV Rao, J Barhen, et al, On computing mobile agent routes for data fusion in distributed sensor networks. IEEE Trans. Knowl. Data Eng. 16(6), 740-753 (2004)

21. M Chen, S Gonzalez, Y Zhang, VCM Leung, Multi-agent itinerary planning for wireless sensor networks. Lecture Notes Inst. Comput. Sci. Soc. Informatics Telecommun. Eng. 22, 584-597 (2009)

22. W Cai, M Chen, T Hara, L Shu, in Proceeding of the 5th Annual International Wireless Internet Conference (WICON). Ga-mip: Genetic algorithm based multiple mobile agents itinerary planning in wireless sensor network, (Singapore, 2010)

23. A Mpitziopoulos, D Gavalas, C Konstantopoulos, G Pantziou, CBID: a scalable method for distributed data aggregation in WSNs. Int. J. Distributed Sensor Netw. 2010(206517), 13 (2010)

24. N SQ, Scalable Network Technologies. Inc. Available: www.qualnet.com. (2011)

25. WR Heinzelman, A Chandrakasan, $\mathrm{H}$ Balakrishnan, in IEEE Proceedings of the 33rd Annual Hawaii International Conference on System Sciences(HICSS-33). Energy-efficient communication protocol for wireless microsensor networks, (Maui, Hawaii, USA, 2000), pp. 1-10. http:// ieeexplore.ieee.org/xpl/articleDetails.jsp?arnumber $=926982$

26. R Malhotra, R Aggarwal, S Monga, Analyzing core migration protocol wireless ad hoc networks. Int. J. Comput. Appl. 9(3), 35-41 (2010)

27. CE Nugraheni, Formal verification of ring-based leader election protocol using predicate diagrams. Int. J. Comput. Sci. Netw. Secur. 9(8), 1-8 (2009)

28. IF Akyildiz, T Melodia, K Chowdhury, A survey on wireless multimedia sensor networks. Int. J. Comput. Telecommun. Netw. 4, 921-960 (2007)

29. B Chen, HH Cheng, J Palen, Mobile-C: a mobile agent platform for mobile $\mathrm{C}(\mathrm{C}++)$ agents. Software-Prac. Exp. 15, 1711-1733 (2006)

30. A Galstyan, B Krishnamachari, K Lerman, in Workshop on Sensor Networks at the 19th National Conference on Artificial Intelligence (AAAI-04). Resource allocation and emergent coordination in wireless sensor networks, (San Jose, California, USA, 2004), pp. 114-118. http://www.isi.edu/ galstyan/ papers/wsn-RA.pdf

31. YK Xiao, XM Shan, Y Ren, Game theory models for IEEE802.11 dcf in wireless ad hoc networks. IEEE Communications Magazine. 43(3), S22-S26 (2005)

32. C-C Tseng, K-C Chen, Y-J Liang, Z-W Tuanand, in The 65th IEEE Vehicular Technology Conference(VTC2007-Spring). Organizing power efficient 
cluster- based network architectures for wireless ad hoc networks, (Dublin, Ireland, 2007), pp. 114-118. http://ieeexplore.ieee.org/stamp/ stamp.jsp?tp=\&arnumber $=4212464$

33. ZL C, RJ M, W A, in IEEE International Conference on Communications. An integrated data-link energy model for wireless sensor networks, (Paris, France, 2004), pp. 3777-3783. http://ieeexplore.ieee.org/stamp/stamp. jsp?tp=\&arnumber $=1313260$

\section{Submit your manuscript to a SpringerOpen ${ }^{\circ}$} journal and benefit from:

- Convenient online submission

- Rigorous peer review

- Immediate publication on acceptance

- Open access: articles freely available online

- High visibility within the field

- Retaining the copyright to your article

Submit your next manuscript at springeropen.com 